\title{
Front Matter: Volume 9085
}

, "Front Matter: Volume 9085," Proc. SPIE 9085, Sensors and Systems for Space Applications VII, 908501 (20 June 2014); doi: 10.1117/12.2073822

SPIE. Event: SPIE Defense + Security, 2014, Baltimore, MD, United States 


\title{
PROCEEDINGS OF SPIE
}

\section{Sensors and Systems for Space Applications VII}

\author{
Khanh D. Pham \\ Joseph L. Cox \\ Editors
}

5-6 May 2014

Baltimore, Maryland, United States

Sponsored and Published by

SPIE 
The papers included in this volume were part of the technical conference cited on the cover and title page. Papers were selected and subject to review by the editors and conference program committee. Some conference presentations may not be available for publication. The papers published in these proceedings reflect the work and thoughts of the authors and are published herein as submitted. The publisher is not responsible for the validity of the information or for any outcomes resulting from reliance thereon.

Please use the following format to cite material from this book:

Author(s), "Title of Paper," in Sensors and Systems for Space Applications VII, edited by Khanh D. Pham, Joseph L. Cox, Proceedings of SPIE Vol. 9085 (SPIE, Bellingham, WA, 2014) Article CID Number.

ISSN: 0277-786X

ISBN: 9781628410228

Published by

SPIE

P.O. Box 10, Bellingham, Washington 98227-0010 USA

Telephone +1 3606763290 (Pacific Time) · Fax +1 3606471445

SPIE.org

Copyright @ 2014, Society of Photo-Optical Instrumentation Engineers.

Copying of material in this book for internal or personal use, or for the internal or personal use of specific clients, beyond the fair use provisions granted by the U.S. Copyright Law is authorized by SPIE subject to payment of copying fees. The Transactional Reporting Service base fee for this volume is $\$ 18.00$ per article (or portion thereof), which should be paid directly to the Copyright Clearance Center (CCC), 222 Rosewood Drive, Danvers, MA 01923. Payment may also be made electronically through CCC Online at copyright.com. Other copying for republication, resale, advertising or promotion, or any form of systematic or multiple reproduction of any material in this book is prohibited except with permission in writing from the publisher. The CCC fee code is 0277-786X/14/\$18.00.

Printed in the United States of America.

Publication of record for individual papers is online in the SPIE Digital Library.

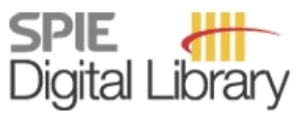

SPIEDigitalLibrary.org

Paper Numbering: Proceedings of SPIE follow an e-First publication model, with papers published first online and then in print and on CD-ROM. Papers are published as they are submitted and meet publication criteria. A unique, consistent, permanent citation identifier (CID) number is assigned to each article at the time of the first publication. Utilization of CIDs allows articles to be fully citable as soon as they are published online, and connects the same identifier to all online, print, and electronic versions of the publication. SPIE uses a six-digit CID article numbering system in which:

- The first four digits correspond to the SPIE volume number.

- The last two digits indicate publication order within the volume using a Base 36 numbering

system employing both numerals and letters. These two-number sets start with 00, 01, 02, 03, 04, $05,06,07,08,09,0 A, 0 B \ldots$. 0Z, followed by 10-1Z, 20-2Z, etc.

The CID Number appears on each page of the manuscript. The complete citation is used on the first page, and an abbreviated version on subsequent pages. Numbers in the index correspond to the last two digits of the six-digit CID Number. 


\section{Contents}

vii Conference Committee

\section{SESSION $1 \quad$ PERVASIVE TECHNOLOGIES SUPPORTING RESPONSIVE SPACE}

908502 Effects of star crossings on the detection of dim objects in orbit and mitigation strategies for improving detection [9085-1]

B. Gessel, S. Cain, Air Force Institute of Technology (United States)

908503 Using ATCOM to enhance long-range imagery collected by NASA's flight test tracking cameras at Armstrong Flight Research Center [9085-2]

A. Paolini, EM Photonics, Inc. (United States); D. Tow, NASA Armstrong Flight Research Ctr. (United States); E. Kelmelis, EM Photonics, Inc. (United States)

908504 FalconSAT-7: a membrane space telescope [9085-3]

G. Andersen, O. Asmolova, T. Dickinson, U.S. Air Force Academy (United States)

908505 Design and operation of SUCHI: the space ultra-compact hyperspectral imager for a small satellite [9085-5]

S. T. Crites, P. G. Lucey, R. Wright, J. Chan, H. Garbeil, K. A. Horton, A. Imai, E. J. Pilger, M. Wood, L. Yoneshige, Univ. of Hawaili at Manoa (United States)

\section{SESSION 2 RADIATION HARDENING AND SPACE WEATHER EFFECTS MITIGATION}

908507 Chalcogenide glass thin-film optics for infrared applications [9085-7]

J. Nath, D. Panjwani, R. E. Peale, Univ. of Central Florida (United States); J. D. Musgraves,

P. Wachtel, J. McKinley, IRradiance Glass, Inc. (United States)

908508 AE9/AP9/SPM: new models for radiation belt and space plasma specification [9085-8] W. R. Johnston, Air Force Research Lab. (United States); T. P. O'Brien, The Aerospace Corp. (United States); G. P. Ginet, MIT Lincoln Lab. (United States); S. L. Huston, Atmospheric and Environmental Research, Inc. (United States); T. B. Guild, The Aerospace Corp. (United States); J. A. Fennelly, Air Force Research Lab. (United States)

\section{SESSION 3 DUAL-USE CIVIL-MILITARY SENSORS AND SYSTEMS I}

9085 0A Development of a spherical aerial vehicle for urban search [9085-10]

K. Hou, H. Sun, Q. Jia, Y. Zhang, Beijing Univ. of Posts and Telecommunications (China)

9085 OB Using atmospheric polarization patterns for Azimuth sensing [9085-1 1]

A. Lompado, T. M. Aycock, Polaris Sensor Technologies, Inc. (United States); B. M. Wheeler, Naval Surface Warfare Ctr. Dahlgren Div. (United States) 
9085 0C Membrane based thermoelectric sensor array for space debris detection [9085-12] F. Haenschke, E. Kessler, A. Ihring, Institut für Photonische Technologien e.V. (Germany); K. D. Bunte, C. Herbst, etamax space GmbH (Germany); M. Mohaupt, Fraunhofer-Institut für Angewandte Optik und Feinmechanik (Germany); T. Fichna, Technische Univ. Braunschweig (Germany); D. Hagedorn, Physikalisch-Technische Bundesanstalt (Germany); H.-G. Meyer, Institut für Photonische Technologien e.V. (Germany)

9085 OE Constrained orbital intercept-evasion [9085-14]

A. Zatezalo, Scientific Systems Co., Inc. (United States); D. Stipanović, Univ. of Illinois at Urbana-Champaign (United States); R. K. Mehra, Scientific Systems Co., Inc. (United States); K. Pham, Air Force Research Lab. (United States)

9085 OF The art and science of missile defense sensor design [9085-15]

B. K. McComas, Raytheon Missile Systems (United States)

SESSION 4 RESILIENT AND SECURE ARCHITECTURES AND PROCESSES FOR DUAL MILITARY-CIVIL SPACE OPERATIONS

90850 Ol SecureCPS: defending a nanosatellite cyber-physical system [9085-18] L. Forbes, H. Vu, Global InfoTek, Inc. (United States); B. Udrea, H. Hagar, Embry-Riddle Aeronautical Univ. (United States); X. D. Koutsoukos, Vanderbilt Univ. (United States); M. Yampolskiy, Vanderbilt Univ. (United States) and Univ. of South Alabama (United States)

9085 oJ A resilient and secure software platform and architecture for distributed spacecraft [9085-19]

W. R. Otte, A. Dubey, G. Karsai, Vanderbilt Univ. (United States)

9085 OK Cyber threat impact assessment and analysis for space vehicle architectures [9085-20] R. M. McGraw, M. J. Fowler, RAM Labs. (United States); D. Umphress, Auburn Univ. (United States); R. A. MacDonald, RAM Labs. (United States)

9085 OL Building space operations resiliency with a multi-tier mission architecture [9085-21] J. Straub, The Univ. of North Dakota (United States)

9085 OM An adaptive process-based cloud infrastructure for space situational awareness applications [9085-22]

B. Liu, Y. Chen, Binghamton Univ. (United States); D. Shen, G. Chen, Intelligent Fusion Technology, Inc. (United States); K. Pham, E. Blasch, B. Rubin, Air Force Research Lab. (United States)

9085 ON Securing resource constraints embedded devices using elliptic curve cryptography [9085-23]

T. Tam, M. Alfasi, M. Mozumdar, California State Univ., Long Beach (United States) 
908500 Simulation study of unmanned aerial vehicle communication networks addressing bandwidth disruptions [9085-24]

S. Wei, L. Ge, W. Yu, Towson Univ. (United States); G. Chen, Intelligent Fusion Technology, Inc. (United States); K. Pham, E. Blasch, Air Force Research Lab. (United States); D. Shen, Intelligent Fusion Technology, Inc. (United States); C. Lu, Towson Univ. (United States)

9085 OP Dynamic autonomous routing technology for IP-based satellite ad hoc networks [9085-25] X. Wang, Foresight Wireless, LLC (United States); J. Deng, Univ. of North Carolina at Greensboro (United States); T. Kostas, G. Rajappan, Foresight Wireless, LLC (United States)

$90850 Q \quad$ Toward effectiveness and agility of network security situational awareness using moving target defense (MTD) [9085-26]

L. Ge, W. Yu, Towson Univ. (United States); D. Shen, G. Chen, Intelligent Fusion Technology, Inc. (United States); K. Pham, E. Blasch, Air Force Research Lab. (United States); C. Lu, Towson Univ. (United States)

\section{SESSION 6 CONNECTIVITY AND DISSEMINATION FOR SPACE APPLICATIONS II}

9085 OS Quantum technology for aerospace applications [9085-28]

B. Jia, Intelligent Fusion Technology, Inc. (United States); K. Pham, Air Force Research Lab. (United States); G. Chen, D. Shen, Z. Wang, G. Wang, Intelligent Fusion Technology, Inc. (United States); E. Blasch, Air Force Research Lab. (United States)

9085 OT Polarization tracking for quantum satellite communications [9085-29]

G. Wang, D. Shen, G. Chen, Intelligent Fusion Technology, Inc. (United States); K. Pham, E. Blasch, Air Force Research Lab. (United States)

9085 OU Quantum key distribution for security guarantees over QoS-driven 3D satellite networks [9085-30]

P. Wang, X. Zhang, Texas A\&M Univ. (United States); G. Chen, Intelligent Fusion Technology, Inc. (United States); K. Pham, E. Blasch, Air Force Research Lab. (United States)

9085 OV Resident space object tracking using an interacting multiple model mixing scheme [9085-31]

Q. M. Lam, LexerdTek Corp. (United States)

\section{SESSION 7 DUAL-USE CIVIL-MILITARY SENSORS AND SYSTEMS II}

9085 0W Towards large scale multi-target tracking [9085-32]

B.-N. Vo, B.-T. Vo, Curtin Univ. (Australia); S. Reuter, Univ. Ulm (Germany); Q. Lam, Lexerdtek Corp. (United States); K. Dietmayer, Univ. Ulm (Germany)

$90850 X \quad$ Space object surveillance using incidental measurements from vehicle-board sensors [9085-33]

S. Roy, Washington State Univ. (United States) 
9085 OY A nano-satellite to study the Sun and the Earth [9085-34]

M. Meftah, A. Irbah, A. Hauchecorne, L. Damé, A. Sarkissian, P. Keckhut, Univ. Versailles St-Quentin, LATMOS-IPSL, CNRS (France); P.-O. Lagage, Commissariat à l'Énergie Atomique (France); S. Dewitte, A. Chevalier, Royal Meteorological Institute of Belgium (Belgium)

$90850 Z$ Dexterous and expedient approach strategies considering non-zero eccentricity orbits and $\mathrm{J}_{2}$ perturbations [9085-35]

C. Remeikas, Y. Xu, Univ. of Central Florida (United States); K. Pham, Air Force Research Lab. (United States); G. Chen, B. Jia, D. Shen, Intelligent Fusion Technology, Inc.

(United States)

908510 Low-complexity image compression with scalable quality control [9085-36]

B. H. Pillman, M. E. Napoli, Exelis Geospatial Systems (United States)

Author Index 


\title{
Conference Committee
}

\author{
Symposium Chair \\ David A. Whelan, Boeing Defense, Space, and Security \\ (United States) \\ Symposium Co-chair
}

Nils R. Sandell Jr., Strategic Technology Office, DARPA (United States)

Conference Chairs

Khanh D. Pham, Air Force Research Laboratory (United States)

Joseph L. Cox, Missile Defense Agency (United States)

\section{Conference Program Committee}

Lisa Belodoff, LightWorks Optics, Inc. (United States)

Thomas George, Zyomed Corporation (United States)

Richard T. Howard, NASA Marshall Space Flight Center (United States)

Ou Ma, New Mexico State University (United States)

Tien M. Nguyen, Raytheon Company (United States)

Andre Samberg, Sec-Control Finland Ltd. (Finland)

Henry Zmuda, University of Florida (United States)

Session Chairs

1 Pervasive Technologies Supporting Responsive Space

Khanh D. Pham, Air Force Research Laboratory (United States)

2 Radiation Hardening and Space Weather Effects Mitigation

Khanh D. Pham, Air Force Research Laboratory (United States)

3 Dual-Use Civil-Military Sensors and Systems I

Khanh D. Pham, Air Force Research Laboratory (United States)

4 Resilient and Secure Architectures and Processes for Dual MilitaryCivil Space Operations

Khanh D. Pham, Air Force Research Laboratory (United States)

5 Connectivity and Dissemination for Space Applications I

Khanh D. Pham, Air Force Research Laboratory (United States)

Genshe Chen, Intelligent Fusion Technology, Inc. (United States) 
6 Connectivity and Dissemination for Space Applications II Khanh D. Pham, Air Force Research Laboratory (United States) Genshe Chen, Intelligent Fusion Technology, Inc. (United States)

7 Dual-Use Civil-Military Sensors and Systems II

Quang M. Lam, LexerdTek Corporation (United States)

Sandip Roy, Washington State University (United States) 